\section{Summary}

Endogenous interferon, a marker of bad pronostic in Argentine haemorrhage fever

Argentine hemorrhagic fever (AHF) is an acute, systemic viral disease caused by Junin virus, a member of the group of arenaviruses. AHF affects mainly young rural workers from an agricultural region known as the humid pampa. The main signs and symptoms during the acute phase of illness are fever, fatigue, malaise, leukopenia, thrombocytopenia (with hemorrhagic manifestations), and neurologic and renal involvment. The mortality is high (20\%-30\%), but it is reduced to $1 \%-3 \%$ when immune plasma is given within eight days after the onset of symptoms. High titers of circulating interferonalpha (IFN- $\alpha$ ) were present in the serum samples taken before treatment, generally between 1,000 and $64,000 \mathrm{IJ} / \mathrm{ml}$. Thus far, these are the highest levels of circulating IFN detected in any human viral disease. IFN titers drastically dropped after treatment with immune plasma. In untreated patients, during the second week of evolution, the IFN titers were significantly higher in fatal cases than in survivors presenting a pronostic value and supporting the hypothesis of a possible deletereous effect. The very high levels of IFN induced by the viral infection, although apparently ineficient as an antiviral agent, are biologically active, inducing a specific marker : 2-5̆ synthetase. The mechanism of in vivo IFN induction by Junin virus is unknown.

\section{ADRESSES}

R. Falcoff : directeur de recherche à l'Inserm. Inserm U. 196, Institut Curie, section de biologie, 26, rue d'Ulm, 75231 Paris Cedex 05. France.

J. Maiztegui : directeur de l'Instituto National. Instituto nacional de estudios sobre virosis hemorragicas, Casilla de Correo 195, 2700 Pergamino, Argentine.

\section{TIRÉS A PART}

R. Falcoff.

\title{
L'interféron endogène, facteur de mauvais pronostic dans la fièvre hémorragique argentine
}

\author{
Rébéca Falcoff, Julio Maiztegui
}

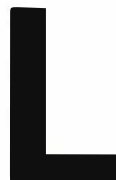

'obtention, en 1980, du premier interféron (IFN) recombinant (interféron $\alpha-2)$ avait rendu possibles des essais en thérapeutique humaine avec cette molécule, essais dont le développement était jusqu'alors limité du fait des quantités réduites d'interféron naturel disponible. Il était clair qu'en raison de ses propriétés essentielles (action antivirale et action anticroissance), deux domaines devaient faire rapidement l'objet de protocoles expérimentaux : les maladies virales et la cancérologie.

En ce qui concerne les premières, nous pensions disposer d'un bon modèle pour démontrer les propriétés antivirales de l'interféron : la fièvre hémorragique argentine (FHA), maladie aiguë, grave et endémique présentant un pic saisonnier régulier et atteignant quelques centaines de personnes chaque année [1]. De plus, l'existence d'un centre hospitalier spécialisé au coeur de la région où sévissait la maladie devait faciliter l'application d'un protocole, géographiquement limité. Les résultats pourraient être obtenus rapidement et le succès thérapeutique évalué solidement. La réalité se révéla toute différente: non seulement nous n'avons pas pu traiter ces patients par l'IFN ; bien au contraire, nous nous sommes trouvés face à une maladie dont la symptomatologie est, de toute évidence, associée à la présence d'interféron endogène.

La FHA a été décrite pour la première fois il y a plus de trente ans et son agent étiologique défini dès
1958. Il s'agit du virus Junin, du nom de la ville argentine dans laquelle les premiers cas sont apparus. Le virus Junin fait partie des Arena virus, famille de virus à ARN dont trois autres sont également pathogènes pour l'homme: le virus Machupo, agent de la fièvre hémorragique bolivienne, le virus Lassa, responsable de la fièvre du même nom sur le continent africain, et le virus de la chorioméningite lymphocytaire présente, comme le rongeur qui est son hôte habituel, dans le monde entier. Les rongeurs autochtones représentent, en revanche, les réservoirs des virus Junin, Machupo et Lassa.

La région endémique de la FHA est située dans la pampa humide, centre céréalier le plus riche du pays. En 1958, lors de la première épidémie reconnue, sa superficie était de $16000 \mathrm{~km}^{2}$; elle est actuellement dix fois plus étendue. L'épidémie est survenue chaque année et, entre 1958 et 1982, plus de 21000 cas ont été enregistrés [2].

La distribution saisonnière de la maladie est liée à la variation dans le nombre de rongeurs, laquelle dépend de leur cycle de reproduction ; le plus grand nombre de rongeurs apparaît à la fin de l'été et en automne. Comme cette période de l'année coïncide avec les récoltes de maïs, de tournesol et de soja, la population à risque est principalement constituée par les travailleurs ruraux. De fait, l'incidence majeure est observée chèz ces travailleurs, de sexe masculin, âgés de 15 à 60 ans. Le virus peut également infecter femmes et enfants. 
Les rongeurs hôtes du virus Junin appartiennent à la famille des Cricétidés. Ils sont infectés chroniquement et ne présentent pas de signes apparents de la maladie; le virus est présent dans différents organes et les taux de virus les plus élevés ont été trouvés dans la salive, laquelle constitue la voie de contamination la plus importante. Le mécanisme de la contagion humaine est mal connu. Il est probable que les voies d'entrée soient le système respiratoire par inhalation d'aérosols infectés, les muqueuses orales ou oculaires ou encore des égratignures au niveau de la peau. La FHA n'est pas contagieuse de personne à personne. Il n'y a pas de vecteur intermédiaire entre le rongeur et l'homme.

\section{Tableau clinique}

La période d'incubation est de 6 à 14 jours. Le début de la maladie est mal défini et sans symptômes spécifiques: fatigue, céphalées, anorexie auxquelles viennent s'ajouter progressivement douleurs musculaires, pertes de l'équilibre, nausées, vomissements et fièvre. Le visage devient érythémateux et l'on observe un œedème palpébral. L'apparition de pétéchies est presque constante dans les régions axillaires et le tiers supérieur du bras. Les muqueuses nasales et buccales sont rouges, congestionnées et les gencives saignent facilement. Des adénopathies sous-cutanées apparaissent. Les malades ne présentent pas de troubles respiratoires, ce qui différencie la FHA de la grippe avec laquelle elle partage des symptômes communs au cours des premiers jours.

Plus tard, tous ces divers symptômes s'accentuent. Des manifestations neurologiques apparaissent et parfois des hémorragies sévères. Les formes neurologiques graves se traduisent par une confusion mentale, une excitation psychomotrice, des tremblements, un délire puis un coma, précédé de convulsions généralisées. Les formes cliniques graves représentent 20 à $30 \%$ de malades non traités et l'issue est généralement fatale dans un état de choc. Dans les formes plus bénignes (70 à $80 \%$ ) et dans la majorité des cas traités précocement avec des doses adéquates de

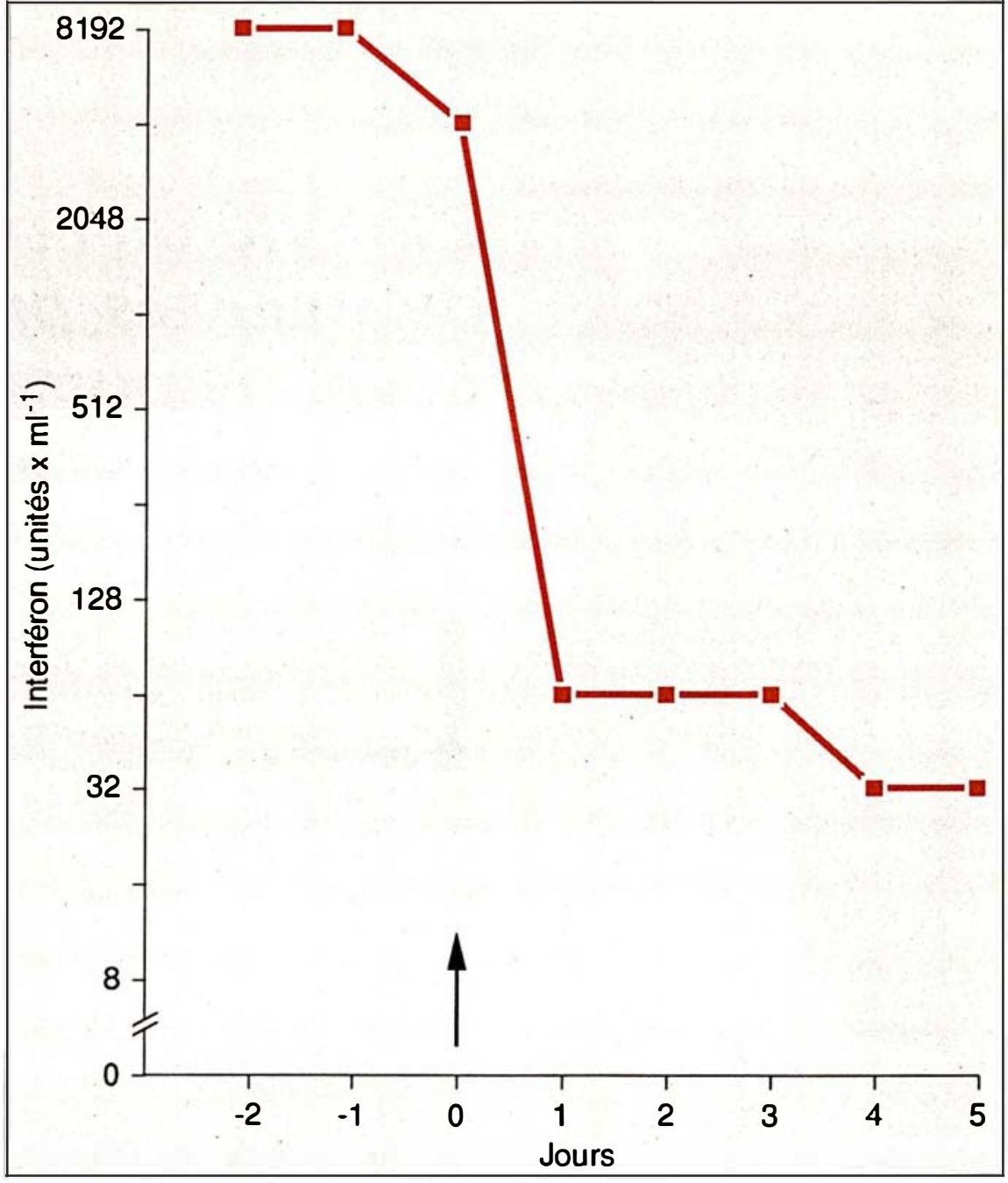

Figure 1. Titres d'interféron dans le sérum d'un patient (n' 4767). Titres mesurés avant et après (i) traitement par du plasma immun.

une nette amélioration s'amorce entre les $8^{\mathrm{e}}$ et $10^{\mathrm{e}}$ jours. La convalescence est, en général, très lente avec asthénie, chute transitoire des cheveux et troubles de la mémoire. La récupération est totale au bout de deux à trois mois et les séquelles sont rares.

\section{Physiopathologie}

Le virus Junin est lymphotrope, sa réplication a probablement lieu dans les organes lymphatiques où les titres de virus les plus élevés ont été observés. Pendant la période aiguë fébrile, la virémie a été démontrée, à des titres très faibles, soit par injection intracérébrale de sang total à des souriceaux nouveau-nés, soit par coculture de la fraction lymphomonocytaire avec des cellules Véro. Néanmoins, l'infection est généralisée et des antigènes viraux ont été mis en évidence dans tous les organes. Le virus a très rarement été isolé dans le système nerveux central, malgré les manifestations neurologiques intervenant au cours de la phase aiguë de la maladie.

La leucopénie et la plaquettopénie font partie des caractéristiques les plus constantes de la FHA, avec des valeurs de 1000 à 2000 globules blancs et 40000 à 90000 plaquettes par $\mathrm{mm}^{3}$. La pathogénie de la diathèse hémorragique n'est pas claire : ni la plaquettopénie ni les altérations des facteurs de la coagulation ne suffisent à l'expliquer [3]. Le virus 
Junin provoque une immunodépression aussi bien humorale qu'à médiation cellulaire [4]. Ceci explique que des infections bactériennes et des mycoses surajoutées soient des complications fréquentes $(20 \%$ des cas). La sous-population des lymphocytes T4 est diminuée et la proportion des lymphocytes T8 augmente, avec pour conséquence une inversion du rapport T4/T8.

\section{Traitement}

Le seul traitement efficace de la FHA est l'administration de plasma immun, obtenu des sujets convalescents [5, 6]. Il a été établi, de façon empirique, que le traitement doit être précoce, c'est-à-dire administré dans les huit jours suivant l'apparition des premiers symptômes et qu'il faut injecter au malade au moins 30000 unités d'anticorps neutralisants par kg de poids corporel. La mortalité est alors réduite à 2-3\%, les manifestations hémorragiques et les surinfections bactériennes diminuent, l'évolution est meilleure. Néanmoins, dans $10 \%$ des cas de FHA traités par le plasma immun, on observe pendant la convalescence, entre quatre et six semaines après la période aiguë, l'apparition d'un syndrome fébrile avec manifestations neurologiques prédominantes cérébelleuses. Ce syndrome neurologique tardif (SNT) se manifeste pendant quelques jours et son évolution est généralement bénigne.

\section{Interféron}

Bien que le plasma de convalescent constitue un traitement efficace de la FHA, il est évident qu'il est loin de constituer le médicament idéal. Sa fourniture est aléatoire car elle dépend de la coopération bénévole des ex-malades. De plus, comme tout dérivé sanguin, chaque préparation doit être testée pour s'assurer de l'absence d'agents infectieux (Junin, Chagas, hépatite, SIDA). Les niveaux d'anticorps neutralisants anti-virus Junin doivent également être déterminés. Enfin, le stockage et la distribution aux centres de traitement ne sont pas des problèmes faciles à résoudre, d'où l'intérêt dans des essais thérapeutiques de molécules antivirales comme les interfé- rons. Mais, avant de décider d'un protocole de cette nature, il fallait déterminer le niveau d'interféron endogène. Les premiers résultats ont été obtenus au cours de l'épidémie de 1982 et ont été complétés durant les années suivantes [7]. Nous avons constaté la présence d'interféron $\alpha$ circulant à des titres très élevés ; après administration de plasma immun, le niveau d'interféron circulant chutait en quelques heures, indépendamment de l'échéance finale (figure 1). Le Tableau I montre les titres d'interféron $\alpha$ circulant de 277 patients, le jour de leur hospitalisation. Il s'agit, en A, de malades pour lesquels l'évolution a été favorable et en $\mathrm{B}$, des cas mortels. Ceux qui ont été hospitalisés moins de huit jours après l'apparition des premiers symptômes ont reçu du plasma immun. Plus tard, le traitement est sans effet, comme nous l'avons dit plus haut. L'interféron est présent chez tous les malades et les titres sont supérieurs à 1000 unités dans $61 \%$ des cas et atteignent jusqu'à 64000 unités. Ce sont les niveaux les plus élevés enregistrés jusqu'ici d'interféron endogène au cours de maladies virales. Les titres couramment trouvés dans la grippe, la rubéole, l'hépatite, etc. ne dépassent pas 500 unités $/ \mathrm{ml}$. A titre comparatif, ajoutons également que l'in- jection de $10 \times 10^{6}$ unités d'interféron $\alpha$, dose couramment utilisée en cancérologie, donne une interféronémie très passagère de 300 à 500 unités $/ \mathrm{ml}$. Le niveau d'interféron chez les patients qui sont hospitalisés dans la phase tardive de la maladie est différent selon l'évolution clinique. Il est significativement plus élevé chez les malades évoluant vers la mort: 17 cas (dont un seul survivant) présentaient encore des titres de 16000-64 000 unités $/ \mathrm{ml}$, c'est-à-dire que les titres très élevés d'interféron ont une valeur pronostique, surtout au cours de la deuxième semaine d'évolution [8]. Nous nous trouvons donc en face d'une situation paradoxale dans laquelle la présence d'une substance antivirale semble nuisible et associé à la pathogénicité du virus Junin. A cet égard, il est intéressant de signaler que le tableau toxicologique dressé au cours des études en phase I avec des doses très élevées d'interféron $\alpha$ $\left(50 \times 10^{6}\right.$ unités) correspond exactement à celui de la FHA : fièvre, fatigue, malaise, leucopénie, thrombocytopénie, perte des cheveux. Ajoutons à ceci le fait que le seul traitement efficace, c'est-à-dire l'administration de plasma immun, a comme conséquence l'effondrement rapide des niveaux d'interféron séri-

\begin{tabular}{|c|c|c|c|}
\hline \multicolumn{4}{|c|}{$\begin{array}{l}\text { Tableau I } \\
\text { NTRE NIVEAUX D'INTERFÉRON SÉRIQUE } \\
\text { UTION CLINIQUE DANS } 277 \text { CAS } \\
\text { RE HÉMORRAGIQUE ARGENTINE }\end{array}$} \\
\hline \multirow{2}{*}{$\begin{array}{l}\text { Jours après } \\
\text { les premiers } \\
\text { symptômes }\end{array}$} & \multicolumn{3}{|c|}{ Titres d'interféron $(\mathrm{U} / \mathrm{ml})^{*}$} \\
\hline & $25-500$ & $1000-8000$ & $16000-64000$ \\
\hline \multicolumn{4}{|c|}{ A : cas à évolution favorable } \\
\hline$\leqslant 8$ & 63 & 95 & 18 \\
\hline$>8$ & 31 & 6 & 1 \\
\hline Total & 94 & 101 & 19 \\
\hline \multicolumn{4}{|l|}{ B : cas mortels } \\
\hline$\leqslant 8$ & 2 & 12 & 13 \\
\hline$>8$ & 12 & 8 & 16 \\
\hline Total & 14 & 20 & 29 \\
\hline
\end{tabular}

$p<0,001$ (test de Student). * Sérums prélevés au moment de l'hospitalisation, avant tout traitement. 
que (figure 1).

Malgré son inefficacité apparente, l'interféron endogène est biologiquement actif. En effet, la 2'-5' oligoadénylate synthétase, enzyme induite in vivo et in vitro par l'interféron, est très fortement augmentée dans les lymphocytes périphériques des patients [9] et elle est toujours présente lorsque les symptômes de la période aiguë ont disparu, ce qui suggère qu'elle n'est pas directement impliquée. Cependant, il est connu que l'interféron induit l'expression d'un certain nombre de gènes [10]. Quelques-uns ont été clonés, mais les propriétés des protéines correspondantes restent inconnues. Il est possible que les effets toxiques de l'interféron puissent être associées à une ou plusieurs de ces protéines. Le mécanisme par lequel l'infection par le virus Junin déclenche la production d'interféron à des niveaux aussi élevés est actuellement encore hypothétique

\section{RÉFÉRENCES}

l. Maiztegui JI. Clinical and epidemiological patterns of argentine hemorrhagic fever. Bull WHO $1975 ; 52$ : 567-75.

2. Rapport du ministère de la Santé Publique Argentine, Buenos Aires, 1984.

3. Molinas FC, Bracco MM, Maiztegui JI. Coagulation studies in argentine hemorraghic fever. J Infect Dis 1981 ; 143 : 1-6.

4. Vallejos DA, Ambrosio AM, Gamboa G, Briggiler AM, Maiztegui JI. Alteraciones de las subpopulaciones linfocitarias en la fiebre hemorragica argentina. Medicina (BsAs) $1985 ; 45: 407$.

5. Maiztegui JI, Fernandez NJ, Damilano AJ. Efficacy of immune plasma in treatment of argentine hemorrhagic fever and association between treatment and late neurological syndrome. Lancet 1979 ; ii (8154) : 1216-7.

6. Enria DA, Briggiler AM, Fernandez NJ, Levis SC, Maiztegui JI. Importance of dose of neutralizing antibodies in treatment of argentine hemorrhagic fever with immune plasma. Lancet 1984; ii (8397) : 255-6.

7. Levis SC, Saavedra MC, Ceccoli C, et al. Endogenous interferon in argentine hemorrhagic fever. J Infect Dis 149: 428-433, 1984. 8. Levis SC, Saavedra MC, Ceccoli C. Correlation between endogenous interferon and the clinical evolution of patients with argentine hemorrhagic fever. J Interferon Res 1985 ; 5: 383-9.

9. Ferbus D, Saavedra MC, Levis S, Maiztegui JI, Falcoff R. High levels of 2'-5' oligoadenylate synthetase in leukocytes from patients with argentine hemorrhagic fever. Relation with endogenous interferon. J Infect Dis 1989 (sous presse).

10. Revel M, Chebath J. Interferon-activated genes. Trends Biochem Sci 1986; 11 : 166-70. 